Stable determination of an inclusion for a class of anisotropic conductivities

This content has been downloaded from IOPscience. Please scroll down to see the full text.

2017 Inverse Problems 33095003

(http://iopscience.iop.org/0266-5611/33/9/095003)

View the table of contents for this issue, or go to the journal homepage for more

Download details:

IP Address: 131.175.161.12

This content was downloaded on 04/08/2017 at 12:26

Please note that terms and conditions apply.

You may also be interested in:

Lipschitz stability for the inverse conductivity problem for a conformal class of anisotropic conductivities

Romina Gaburro and Eva Sincich

Uniqueness and Lipschitz stability of an inverse boundary value problem for time-harmonic elastic waves

Elena Beretta, Maarten V de Hoop, Elisa Francini et al.

Lipschitz continuous dependence of piecewise constant Lamé coefficients from boundary data: the case of non-flat interfaces

Elena Beretta, Elisa Francini, Antonino Morassi et al.

Size estimates of an obstacle in a stationary Stokes fluid

E Beretta, C Cavaterra, J H Ortega et al.

Stable determination of cavities in elastic bodies

Antonino Morassi and Edi Rosset

Lipschitz stability for the inverse Robin problem

E Sincich

Stable determination of an immersed body in a stationary Stokes fluid

Andrea Ballerini 


\title{
Stable determination of an inclusion for a class of anisotropic conductivities
}

\author{
Michele Di Cristo ${ }^{1}$ and Yong Ren \\ Politecnico di Milano, Milano, Italy \\ E-mail: michele.dicristo@polimi.it and yong.ren@polimi.it
}

Received 1 March 2017, revised 19 June 2017

Accepted for publication 26 June 2017

Published 1 August 2017

CrossMark

\begin{abstract}
We deal with the inverse problem of determining an inclusion within an electrical conductor from electrostatic boundary measurements. We consider an anisotropic conductivity and provide logarithmic type stability.

Keywords: stability estimates, inclusion determination, anisotropic conductivity
\end{abstract}

\section{Introduction}

In this paper we deal with the problem of determining an inclusion $D$ in an electrical conductor $\Omega$. Particularly, we are interested in analysing anisotropic bodies. The region $D$ represents a portion of $\Omega$ where the conductivity $\sigma$ has a jump of discontinuity across the interface. Denoted by $A(x)$ the known matrix conductivity of $\Omega$, and $k A(x)$ the conductivity inside of $D$, where $k$ is an unknown function. Prescribing a voltage $f \in H^{1 / 2}(\partial \Omega)$ on the boundary $\partial \Omega$ of the domain, the induced potential $u \in H^{1}(\Omega)$ is the solution of the boundary value problem

$$
\begin{cases}\operatorname{div}\left(\left(A(x)+(k-1) A(x) \chi_{D}\right) \nabla u\right)=0 & \text { in } \Omega \\ u=f & \text { on } \partial \Omega,\end{cases}
$$

where $\chi_{D}$ is the characteristic function of the set $D$. Our inverse problem is addressed to determine the anomalous region $D$ when the Dirichlet-to-Neumann map $\Lambda_{D}$

$$
\begin{aligned}
\Lambda_{D}: H^{1 / 2}(\partial \Omega) & \longrightarrow H^{-1 / 2}(\partial \Omega) \\
f & \longrightarrow \frac{\partial u}{\partial \nu} \mid \partial \Omega
\end{aligned}
$$

is given for any $f \in H^{1 / 2}(\partial \Omega)$. Here, $\nu$ denotes the outer unit normal to $\partial \Omega$, and $\frac{\partial u}{\partial \nu} \mid \partial \Omega$ corresponds to the current density measured on $\partial \Omega$. Thus, the Dirichlet-to-Neumann map represents the knowledge of infinitely many boundary measurements.

${ }^{1}$ Author to whom any correspondence should be addressed. 
This problem is considered by Isakov [Is88], where uniqueness for isotropic conductivities is established. Stability is obtained in [Al-DC] for piecewise constant conductivities and is extended in [DC] with conductivities of the form $\sigma(x)=a(x)+b(x) \chi_{D}$, where $a$ is a $C^{1, \alpha}$ function and $b$ is a $C^{\alpha}$ unknown function. Concerning the anisotropic case, not many results are available. The uniqueness result is proven in $[\mathrm{Kw}]$ with conductivities of the form $\sigma=A+(B-A) \chi_{D}$, where $A, B$ are matrix valued functions.

In this paper we analyse the stability, given the reasonable a priori information on the unknown, the inclusion depends continuously on the Dirichlet-to-Neumann map with modulus of continuity of logarithmic type. Let us stress that this rate of continuity is optimal as it is shown by examples in [DC-Ro].

The argument follows the lines of the isotropic case presented in [Al-DC] (see also [DC2] for the inverse scattering case, [DC-Ve], [DC-Ve2] for the thermal imaging case and [Al-DCMo-Ro] for elasticity) and it is based on the use of singular solutions and quantitative estimates of unique continuation. We will carry on an accurate analysis of singular solutions by studying the asymptotic behaviour when the singularity gets close to the interface $\partial D$. Combining this with a control of boundary smallness propagation, we will get the stability estimates. This is the first analysis to study the stability for more general conductivities of the form $A+(B-A) \chi_{D}$. It seems reasonable that this argument also works in this case, but a deeper analysis on the asymptotic behaviour of the fundamental solutions (theorem 4.1) is needed.

The paper is organised as follows. In the next section 2 , after some notations and definitions, we will state our main result, whose proof is presented in section 3 . The proof is based on some auxiliary propositions proven in section 4 .

\section{Main result}

Let us first premise some notations and definitions. For points $x \in \mathbb{R}^{n}$, we will write $x=\left(x^{\prime}, x_{n}\right)$, where $x^{\prime} \in \mathbb{R}^{n-1}$ and $x_{n} \in \mathbb{R}$. Moreover, denoted by dist $(\cdot, \cdot)$ the standard Euclidean distance, we define

$B_{r}(x)=\left\{y \in \mathbb{R}^{n} \mid \operatorname{dist}(x, y) \leqslant r\right\}, \quad B_{r}^{\prime}\left(x^{\prime}\right)=\left\{y^{\prime} \in \mathbb{R}^{n-1} \mid \operatorname{dist}\left(x^{\prime}, y^{\prime}\right) \leqslant r\right\}$

as the open balls with radius $r$ centered at $x$ and $x^{\prime}$ respectively. We write $Q_{r}(x)=$ $B_{r}^{\prime}\left(x^{\prime}\right) \times\left(x_{n}-r, x_{n}+r\right)$ for the cylinder in $\mathbb{R}^{n}$. For simplicity, we use $B_{r}, B_{r}^{\prime}, Q_{r}$ instead of $B_{r}(0), B_{r}^{\prime}\left(0^{\prime}\right)$ and $Q_{r}(0)$ respectively. We shall also denote half domain, as well as its associated ball and cylinder

$$
\mathbb{R}_{+}^{n}=\left\{\left(x^{\prime}, x_{n}\right) \in \mathbb{R}^{n} \mid x_{n}>0\right\} ; B_{r}^{+}=B_{r} \cap \mathbb{R}_{+}^{n} ; Q_{r}^{+}=Q_{r} \cap \mathbb{R}_{+}^{n} .
$$

Definition 2.1. Let $\Omega$ be the bounded domain in $\mathbb{R}^{n}$. We say a portion $S$ of $\partial \Omega$ is of Lipschitz class with constants $r, L>0$ if for any point $p \in S$, there exists a rigid transformation $\varphi: \mathbb{R}^{n-1} \mapsto \mathbb{R}$ of coordinates under which we have $p=0$ and

$$
\Omega \cap B_{r}=\left\{\left(x^{\prime}, x_{n}\right) \in B_{r} \mid x_{n}>\varphi\left(x^{\prime}\right)\right\}
$$

where $\varphi(\cdot)$ is a Lipschitz continuous function on $B_{r}^{\prime}$, which satisfies

$$
\varphi(0)=0
$$

and

$$
\|\varphi\|_{C^{0,1}\left(B_{r}^{\prime}\right)} \leqslant L r
$$


We shall say that $\Omega$ is of Lipschitz class with constants $r, L$ if $\partial \Omega$ is of Lipschitz class with the same constants.

Definition 2.2. Let $\Omega$ be the bounded domain in $\mathbb{R}^{n}$. Given $\alpha \in(0,1]$, we say a portion $S$ of $\partial \Omega$ is of $C^{1, \alpha}$ class with constants $r, L>0$ if for any point $p \in S$, there exists a rigid transformation $\varphi: \mathbb{R}^{n-1} \mapsto \mathbb{R}$ of coordinates under which we have $p=0$ and

$$
\Omega \cap B_{r}=\left\{\left(x^{\prime}, x_{n}\right) \in B_{r} \mid x_{n}>\varphi\left(x^{\prime}\right)\right\},
$$

where $\varphi(\cdot)$ is a $C^{1, \alpha}$ function on $B_{r}^{\prime}$, which satisfies

$$
\varphi(0)=|\nabla \varphi(0)|=0
$$

and

$$
\|\varphi\|_{C^{1, \alpha}\left(B_{r}^{\prime}\right)} \leqslant L r
$$

where the norm is defined as

$$
\begin{aligned}
& \|\varphi\|_{C^{1, \alpha}\left(B_{r}^{\prime}\right)}:=\|\varphi\|_{L^{\infty}\left(B_{r}^{\prime}\right)}+r\|\nabla \varphi\|_{L^{\infty}\left(B_{r}^{\prime}\right)}+r^{1+\alpha}|\nabla \varphi|_{\alpha, B_{r}^{\prime}} \\
& |\nabla \varphi|_{\alpha, B_{r}^{\prime}}:=\sup _{\substack{x^{\prime}, y^{\prime} \in B_{r}^{\prime} \\
x^{\prime} \neq y^{\prime}}} \frac{\left|\nabla \varphi\left(x^{\prime}\right)-\nabla \varphi\left(y^{\prime}\right)\right|}{\left|x^{\prime}-y^{\prime}\right|} .
\end{aligned}
$$

\subsection{Assumptions and a priori data}

Given constants $r_{0}, M_{0}, M_{1}, \delta_{0}, \bar{A}, \lambda>0$ and $0<\alpha<1$, we assume the domain $\Omega \subset \mathbb{R}^{n}$ is bounded

$$
|\Omega| \leqslant M_{1} r_{0}^{n}
$$

where $|\cdot|$ denotes the Lebesgue measure.

The inclusion $D$ is assumed to stay away from the boundary of the domain, as $\operatorname{dist}(D, \partial \Omega) \geqslant \delta_{0}$, and also $\Omega \backslash D$ is connected. Both $\partial \Omega$ and $\partial D$ are of $C^{1, \alpha}$ class with constants $r_{0}, M_{0}$. We shall consider the conductivities

$$
\sigma_{D}(x)=A(x)+A(x)(k-1) \chi_{D}
$$

where $A(x)$ is a known Lipschitz symmetric matrix valued function satisfying $\|A\|_{C^{0,1}(\Omega)} \leqslant \bar{A}$ and ellipticity condition with constant $\lambda>0$ such that

$$
\lambda^{-1}|\xi|^{2} \leqslant A(x) \xi \cdot \xi \leqslant \lambda|\xi|^{2}, \quad \forall x \in \Omega, \xi \in \mathbb{R}^{n} .
$$

We refer to $n, k, r_{0}, M_{0}, M_{1}, \alpha, \delta_{0}, \bar{A}, \lambda$ as the a priori data.

Theorem 2.3. Let $\Omega \subset \mathbb{R}^{n}, n \geqslant 2, k>0, k \neq 1$ be given, and $D_{1}$ and $D_{2}$ be two inclusions in $\Omega$. With the assumptions above, for any given $\varepsilon>0$ if we have

$$
\left\|\Lambda_{D_{1}}-\Lambda_{D_{2}}\right\|_{\mathcal{L}\left(H^{1 / 2}, H^{-1 / 2}\right)}<\epsilon
$$

then

$$
d_{\mathcal{H}}\left(\partial D_{1}, \partial D_{2}\right) \leqslant \omega(\epsilon)
$$


where $\omega$ is an increasing function on $[0,+\infty)$ that satisfies

$$
\omega(t) \leqslant C|\log t|^{-\eta} \quad \forall t \in(0,1)
$$

and $C>0,1 \geqslant \eta>0$ are constants only depending on the a priori data.

\section{Proof of the main result}

The proof of theorem 2.3 is based on some auxiliary propositions, and their proofs are collected in the next section 4. In what follows we define layers of our domains. We denote by $\mathcal{G}$ the connected component of $\Omega \backslash\left(D_{1} \cup D_{2}\right)$, whose boundary contains $\partial \Omega, \Omega_{D}=\Omega \backslash \overline{\mathcal{G}}, \Omega_{r}:=\{x \in \mathcal{C} \Omega \mid \operatorname{dist}(x, \Omega)>r\}, S_{2 r}:=\left\{x \in \mathbb{R}^{n} \mid r \leqslant \operatorname{dist}(x, \Omega) \leqslant 2 r\right\}$ and $\mathcal{G}^{h}:=\left\{x \in \mathcal{G} \mid \operatorname{dist}\left(x, \Omega_{D}\right) \geqslant h\right\}$.

We introduce a variation of the Hausdorff distance called the modified distance, which can simplify our proof.

Definition 3.1. The modified distance between $D_{1}$ and $D_{2}$ is defined as

$$
d_{m}\left(D_{1}, D_{2}\right):=\max \left\{\sup _{x \in \partial \Omega_{D} \cap \partial D_{1}} \operatorname{dist}\left(x, \partial D_{2}\right), \sup _{x \in \partial \Omega_{D} \cap \partial D_{2}} \operatorname{dist}\left(x, \partial D_{1}\right)\right\} .
$$

We remark here that $d_{m}$ is not a metric, and in general, it does not dominate the Hausdorff distance. However, under our a priori assumptions on the inclusion, the following lemma holds.

Lemma 3.2. Under the assumptions of theorem 2.3, there exists a constant $c_{0} \geqslant 1$ only depending on $M_{0}$ and $\alpha$ such that

$$
d_{\mathcal{H}}\left(\partial D_{1}, \partial D_{2}\right) \leqslant c_{0} d_{m}\left(D_{1}, D_{2}\right) .
$$

Proof. See [Al-DC], proposition 3.3].

Another obstacle comes from the fact that the propagation of smallness arguments are based on an iterated application of the three spheres inequality for solutions of the equation over chains of balls contained in $\mathcal{G}$. Therefore, it is crucial to control from below the radii of these balls. In the following lemma 3.3 we treat the case of points of $\partial \Omega_{D}$ that are not reachable by such chains of balls. This problem was originally considered by [Al-Si] in the context of cracks detection in electrical conductors.

Let us premise some notations. Given $O=(0, \ldots, 0)$ the origin, $v$ a unit vector, $H>0$ and $\vartheta \in\left(0, \frac{\pi}{2}\right)$, we denote

$$
C(O, v, H, \vartheta)=\left\{x \in \mathbf{R}^{n}:|x-(x \cdot v) v| \leqslant \sin \vartheta|x|, 0 \leqslant x \cdot v \leqslant H\right\}
$$

the closed truncated cone with vertex at $O$, axis along the direction $v$, height $H$ and aperture $2 \vartheta$. Given $R, d, 0<R<d$ and $Q=-d e_{n}$, where $e_{n}=(0, \ldots, 0,1)$, let us consider the cone $C\left(O,-e_{n}, \frac{d^{2}-R^{2}}{d}, \arcsin \frac{R}{d}\right)$.

From now on, without loss of generality, we assume that

$$
d_{m}\left(D_{1}, D_{2}\right)=\max _{x \in \partial D_{1} \cap \partial \Omega_{D}} \operatorname{dist}\left(x, \partial D_{2}\right)
$$

and we write $d_{m}=d_{m}\left(D_{1}, D_{2}\right)$. 
Let us define

$$
S_{2 \rho_{0}}=\left\{x \in \mathbf{R}^{n} \mid \rho_{0}<\operatorname{dist}(x, \bar{\Omega})<2 \rho_{0}\right\} .
$$

We shall make use of paths connecting points in order that appropriate tubular neighborhoods of such paths still remain within $\mathbf{R}^{n} \backslash \Omega_{D}$. Let us pick a point $P \in \partial D_{1} \cap \partial \Omega_{D}$, let $\nu$ be the outer unit normal to $\partial D_{1}$ at $P$ and let $d>0$ be such that the segment $[(P+d \nu), P]$ is contained in $\mathbf{R}^{n} \backslash \Omega_{D}$. Given $P_{0} \in \mathbf{R}^{n} \backslash \Omega_{D}$, let $\gamma$ be a path in $\mathbf{R}^{n} \backslash \Omega_{D}$ joining $P_{0}$ to $P+d \nu$. We consider the following neighborhood of $\gamma \cup[(P+d \nu), P] \backslash\{P\}$ formed by a tubular neighborhood of $\gamma$ attached to a cone with vertex at $P$ and axis along $\nu$

$$
V(\gamma)=\bigcup_{S \in \gamma} B_{R}(S) \cup C\left(P, \nu, \frac{d^{2}-R^{2}}{d}, \arcsin \frac{R}{d}\right) .
$$

Note that two significant parameters are associated to such a set, the radius $R$ of the tubular neighborhood of $\gamma, \cup_{S \in \gamma} B_{R}(S)$, and the half-aperture $\arcsin \frac{R}{d}$ of the cone $C\left(P, \nu, \frac{d^{2}-R^{2}}{d}\right.$, $\left.\arcsin \frac{R}{d}\right)$. In other terms, $V(\gamma)$ depends on $\gamma$ and also on the parameters $R$ and $d$. At each of the following steps, such two parameters shall be appropriately chosen and shall be accurately specified. For the sake of simplicity we convene to maintain the notation $V(\gamma)$ also when different values of $R, d$ are introduced. Also we warn the reader that it will be convenient at various stages to use a reference frame such that $P=O=(0, \ldots, 0)$ and $\nu=-e_{n}$.

Lemma 3.3. Under the above notation, there exist positive constants $\bar{d}, c_{1}$, where $\frac{\bar{d}}{\rho_{0}}$ only depends on $M_{0}$ and $\alpha$, and $c_{1}$ only depends on $M_{0}, \alpha, M_{1}$, and there exists a point $P \in \partial D_{1}$ satisfying

$$
c_{1} d_{m} \leqslant \operatorname{dist}\left(P, D_{2}\right)
$$

and such that, giving any point $P_{0} \in S_{2 \rho_{0}}$, there exists a path $\gamma \subset\left(\overline{\Omega^{\rho_{0}}} \cup S_{2 \rho_{0}}\right) \backslash \overline{\Omega_{D}}$ joining $P_{0}$ to $P+\bar{d} \nu$, where $\nu$ is the unit outer normal to $D_{1}$ at $P$, such that, choosing a coordinate system with origin $O$ at $P$ and axis $e_{n}=-\nu$, the set $V(\gamma)$ introduced in (3.2) satisfies

$$
V(\gamma) \subset \mathbf{R}^{n} \backslash \Omega_{D}
$$

provided $R=\frac{\bar{d}}{\sqrt{1+L_{0}^{2}}}$, where $L_{0}, 0<L_{0} \leqslant M_{0}$, is a constant only depending on $M_{0}$ and $\alpha$.

Proof. See [Al-DC-Mo-Ro], lemma 4.2].

In order to use the information provided by the boundary measurements to evaluate the distance between two inclusions $D_{1}$ and $D_{2}$, we apply the following identity firstly introduced by Alessandrini in [Al]. Let $u_{i} \in H^{1}(\partial \Omega), i=1,2$, be solutions to (1.1) with conductivities $\sigma_{D_{i}}=A(x)+(k-1) A(x) \chi_{D_{i}}$ respectively, we have

$$
\int_{\Omega}\left(\sigma_{D_{1}} \nabla u_{1} \cdot \nabla u_{2}\right)-\int_{\Omega}\left(\sigma_{D_{2}} \nabla u_{1} \cdot \nabla u_{2}\right)=\int_{\partial \Omega} u_{1}\left[\Lambda_{D_{1}}-\Lambda_{D_{2}}\right] u_{2} .
$$

For the operator $\operatorname{div}\left(\left(A(x)+A(x)(k-1) \chi_{D_{i}}\right) \nabla \cdot\right)$, denoted by $\Gamma_{D_{i}}, i=1,2$ the associated fundamental solutions. We apply (3.3) to $\Gamma_{D_{1}}$ and $\Gamma_{D_{2}}$ with $y, z$ belonging to the complement set of $\Omega$, obtains 


$$
\begin{aligned}
& \left.\int_{\Omega}\left(A(x)+(k-1) A(x) \chi_{D_{1}}\right) \nabla \Gamma_{D_{1}}(\cdot, y) \cdot \nabla \Gamma_{D_{2}}(\cdot, z)\right) \\
& \left.-\int_{\Omega}\left(A(x)+(k-1) A(x) \chi_{D_{2}}\right) \nabla \Gamma_{D_{1}}(\cdot, y) \cdot \nabla \Gamma_{D_{2}}(\cdot, z)\right) \\
& =\int_{\partial \Omega} \Gamma_{D_{1}}(\cdot, y)\left[\Lambda_{D_{1}}-\Lambda_{D_{2}}\right] \Gamma_{D_{2}}(\cdot, z) .
\end{aligned}
$$

For $y, z \in \mathcal{G} \cup \mathcal{C} \Omega$, where $\mathcal{C} \Omega$ is the complementary of $\Omega$, we define

$$
\begin{aligned}
& S_{D_{1}}(y, z)=(k-1) \int_{D_{1}} A(x) \nabla \Gamma_{D_{1}}(\cdot, y) \cdot \nabla \Gamma_{D_{2}}(\cdot, z) \\
& S_{D_{2}}(y, z)=(k-1) \int_{D_{2}} A(x) \nabla \Gamma_{D_{1}}(\cdot, y) \cdot \nabla \Gamma_{D_{2}}(\cdot, z) \\
& f(y, z)=S_{D_{1}}(y, z)-S_{D_{2}}(y, z) .
\end{aligned}
$$

Therefore (3.4) can be written as

$$
f(y, z)=\int_{\partial \Omega} \Gamma_{D_{1}}(\cdot, y)\left[\Lambda_{D_{1}}-\Lambda_{D_{2}}\right] \Gamma_{D_{2}}(\cdot, z), \quad \forall y, z \in \mathcal{C} \bar{\Omega} .
$$

The following two propositions provide quantitative estimates on $f(y, y)$ and $S_{D_{1}}(y, y)$, when moving $y$ towards $O$ along $\nu(O)$.

Proposition 3.4. Given $\epsilon>0$, the domain $\Omega$ and inclusions $D_{1}, D_{2}$, and let $y=h \nu(O)$, if we have

$$
\left\|\Lambda_{D_{1}}-\Lambda_{D_{2}}\right\|_{L\left(H^{1 / 2}, H^{-1 / 2}\right)}<\epsilon
$$

then for every $h$ where $0<h<c r, 0<c<1$, and $c$ depends on $M_{0}$, we have

$$
|f(y, y)| \leqslant C_{0} \frac{\epsilon^{B h^{F}}}{h^{A}}
$$

here $0<A<1$ and $C_{0}, B, F>0$ are constants that depend only on the a priori data.

Proposition 3.5. Given $\epsilon>0$, the domain $\Omega$ and inclusions $D_{1}, D_{2}$, and let $y=h \nu(O)$ defined as above. Then for every $0<h<h_{0} / 2$

$$
\left|S_{D_{1}}(y, y)\right| \geqslant C_{1} h^{2-n}-C_{2} d_{m}^{2-2 n}+C_{3}
$$

where $h_{0}:=\frac{r}{2} \min \left[\frac{1}{2}\left(8 M_{0}\right)^{-1 / \alpha}, \frac{1}{2}\right]$, and $C_{1}, C_{2}, C_{3}$ are positive constants depending only on the a priori data.

Now, we have all the ingredients to conclude this section with the proof of theorem 2.3.

Proof of theorem 2.3. We start from the origin of the coordinate system, a point $O \in \partial D_{1} \cap \partial \Omega_{D}$, for which the maximum in definition 3.1 is attainted

$$
d_{m}:=d_{m}\left(D_{1}, D_{2}\right)=\operatorname{dist}\left(O, D_{2}\right) .
$$

Then with a transformation of coordinates $y=h \nu(O)$ where $0<h<h_{1}, h_{1}:=\min \left\{d_{m}, c r_{0}\right.$, $\left.h_{0} / 2\right\}, 0<c<1$, where $c$ depends on $M_{0}$. By applying [Al-DC] proposition 3.4 (i), i.e. 
$\left|\nabla_{x} \Gamma_{D_{i}}(x, y)\right| \leqslant c_{1}|x-y|^{1-n}, i=1,2$, where $c_{1}>0$ depending only on $k, n, \alpha, M_{0}$; we have

$$
\begin{aligned}
\left|S_{D_{2}}(y, y)\right| & =(k-1) \int_{D_{2}} A(x) \nabla \Gamma_{D_{1}}(\cdot, y) \nabla \Gamma_{D_{2}}(\cdot, y) \\
& \leqslant(k-1) \bar{A} \int_{D_{2}}\left(c_{1}\left|d_{m}-h\right|^{1-n}\right)^{2} \leqslant C_{4}\left|d_{m}-h\right|^{2-2 n}\left|D_{2}\right|
\end{aligned}
$$

where $|\cdot|$ is the Lebesgue measure and $C_{4}$ depends on $k, n, \alpha, M_{0}, \bar{A}$. From (3.5), we apply the triangular inequality to $f(y, y)$, by its definition, we get

$$
\left|S_{D_{1}}(y, y)\right|-\left|S_{D_{2}}(y, y)\right| \leqslant\left|S_{D_{1}}(y, y)-S_{D_{2}}(y, y)\right|=|f(y, y)| \leqslant C_{0} \frac{\epsilon^{B h^{F}}}{h^{A}} .
$$

Therefore, together with (3.6)-(3.8), we obtain

$$
C_{1} h^{2-n}-C_{2} d_{m}^{2-2 n}+C_{3} \leqslant C_{4}\left|d_{m}-h\right|^{2-2 n}\left|D_{2}\right|+C_{0} \frac{\epsilon^{B h^{F}}}{h^{A}} .
$$

Let $C_{3}=C_{2} d_{m}^{2-2 n}, C_{5}=C_{4}\left|D_{2}\right| / C_{0}$ and $C_{6}=C_{1} / C_{0}$, we have

$$
C_{5}\left|d_{m}-h\right|^{2-2 n} \geqslant C_{6} h^{2-n}-\frac{\epsilon^{B h^{F}}}{h^{A}}=C_{6} h^{2-n}\left(1-\epsilon^{B h^{F}} h^{K}\right)
$$

where $0<K=n-2-A$. Now let $h=h(\epsilon)=\min \left\{|\ln \epsilon|^{-\frac{1}{2 F}}, d_{m}\right\}$, for $0<\epsilon \leqslant \epsilon_{1}, \epsilon_{1} \epsilon$ $(0,1)$ such that $\exp \left(-B\left|\ln \epsilon_{1}\right|^{1 / 2}\right)=1 / 2$. Thanks to lemma 3.2, if $d_{m} \leqslant|\ln \epsilon|^{-\frac{1}{2 F}}$, the main theorem 2.3 is proved by setting $\eta=\frac{1}{2 F}>0$

$$
d_{\mathcal{H}}\left(\partial D_{1}, \partial D_{2}\right) \leqslant c_{0} d_{m} \leqslant c_{0}|\ln \epsilon|^{-\eta}=\omega(\epsilon) .
$$

If $d_{m} \geqslant|\ln \epsilon|^{-\frac{1}{2 F}}$, it is easy to check

$$
\left(d_{m}-h\right)^{2-2 n} \geqslant \frac{C_{5}}{2 C_{6}} h^{2-n} \Longrightarrow d_{m} \leqslant C_{7}|\ln \epsilon|^{-\frac{n-2}{4 F(n-1)}}
$$

here we solve $d_{m}$ because $h=h(\epsilon)=|\ln \epsilon|^{-\frac{1}{2 F}}$, and $C_{7}$ depends only on the a priori data. Therefore we conclude the proof by setting $\eta=\frac{n-2}{4 F(n-1)}$

$$
d_{\mathcal{H}}\left(\partial D_{1}, \partial D_{2}\right) \leqslant c_{0} d_{m} \leqslant c_{0} C_{7}|\ln \epsilon|^{-\eta}=\omega(\epsilon)
$$

and for $\epsilon_{1} \leqslant \epsilon$, we can also conclude the proof because $d_{m} \leqslant \operatorname{diam} \Omega \leqslant M_{1} r_{0}^{n}$.

$$
d_{\mathcal{H}}\left(\partial D_{1}, \partial D_{2}\right) \leqslant c_{0} d_{m} \leqslant c_{0} M_{1} r_{0}^{n}=\omega(\epsilon)
$$

Thus, we can conclude the proof theorem 2.3 by (3.9)-(3.11)

$$
d_{\mathcal{H}}\left(\partial D_{1}, \partial D_{2}\right) \leqslant C d_{m}=\omega(\epsilon)
$$

where $C$ only depends on the a priori data. 


\section{Proofs of propositions 3.4 and 3.5}

Proof of proposition 3.4. Let us consider $f(y, \cdot)$ with a fixed $y \in S_{2 r}$, then

$$
\operatorname{div}\left(A(x) \nabla_{w} f(y, w)\right)=0 \text { in } \mathcal{C} \bar{\Omega}_{D} .
$$

For $w \in S_{2 r}$, by (2.1) and (3.4) and upper bound for $\Gamma_{D_{i}}$ (see [Li-St-We]),

$$
\begin{aligned}
|f(y, w)| & =\left|\int_{\partial \Omega} \Gamma_{D_{1}}(x, y)\left[\Lambda_{D_{1}}-\Lambda_{D_{2}}\right] \Gamma_{D_{2}}(x, w) \mathrm{d} x\right| \\
& \leqslant \int_{\partial \Omega}|x-y|^{2-n} \cdot\left\|\Lambda_{D_{1}}-\Lambda_{D_{2}}\right\| \cdot|x-w|^{2-n} \mathrm{~d} x \\
& =C\left(r, M_{0}\right)\left\|\Lambda_{D_{1}}-\Lambda_{D_{2}}\right\|=\epsilon, \quad y, w \in S_{2 r}
\end{aligned}
$$

we adopt the same notation $\epsilon$ to represent the smallness quantity for the above formula, because (2.1) holds for any given $\epsilon>0$. Now we consider the case when $w \in \mathcal{G}^{h}$, we have the upper bound of $\left|S_{D_{1}}\right|$

$$
\begin{aligned}
\left|S_{D_{1}}(y, w)\right| & =\left|(k-1) \int_{D_{1}} A(x) \nabla \Gamma_{D_{1}}(x, y) \nabla \Gamma_{D_{2}}(x, w) \mathrm{d} x\right| \\
& \leqslant|k-1| \bar{A} \int_{D_{1}}\left|\nabla \Gamma_{D_{1}}(x, y)\right|\left|\nabla \Gamma_{D_{2}}(x, w)\right| \mathrm{d} x \\
& \leqslant c_{0}\left(k, \bar{A}, r, M_{0}\right) \int_{D_{1}}|x-y|^{1-n}|x-w|^{1-n} \mathrm{~d} x \leqslant c_{0} h^{1-n} .
\end{aligned}
$$

Similarly, we have $\left|S_{D_{2}}(y, w)\right| \leqslant c_{0} h^{1-n}$. By definition, we conclude that

$$
|f(y, w)| \leqslant c_{0} h^{1-n}, \quad w \in \mathcal{G}^{h}, y \in S_{2 r} .
$$

Now we apply the three spheres inequality for supremum norms of harmonic function $v$. In our case, for every $x \in \mathcal{G} \cup S_{2 r} \cup \Omega_{r}$, we apply page 781 [Al-Be-Ro-Ve]. We obtain that for every $1<\beta_{1}<\beta_{2}$, there exists $\tau \in(0,1)$ and constant $c_{1}=c_{1}\left(\bar{R}, M_{0}, \beta_{1}, \beta_{2}, n\right)$ such that

$$
\|v\|_{L^{\infty}\left(B_{\beta_{1} \bar{R}}(x)\right)} \leqslant c_{1}\|v\|_{L^{\infty}\left(B_{\bar{R}}(x)\right)}^{\tau} \cdot\|v\|_{L^{\infty}\left(B_{\beta_{2} \bar{R}}(x)\right)}^{1-\tau} .
$$

Now we apply the above inequality by choosing $\beta_{1}=3 / 2, \beta_{2}=2$ and $\bar{R}=r$ for harmonic function $f(y, \cdot)$, we get

$$
\|f(y, \cdot)\|_{L^{\infty}\left(B_{3 r / 2}(x)\right)} \leqslant c_{1}\|f(y, \cdot)\|_{L^{\infty}\left(B_{r}(x)\right)}^{\tau} \cdot\|f(y, \cdot)\|_{L^{\infty}\left(B_{2 r}(x)\right)}^{1-\tau} .
$$

Constructing a chain of balls on an arc connecting $x$ with $w$ for $w \in \mathcal{G}^{h}$, as in [Al-DC, proposition 3.5, page 212], we have, for any $R, 0<R<r$

$$
\|f(y, \cdot)\|_{L^{\infty}\left(B_{R / 2}(w)\right)} \leqslant c_{2} \cdot \epsilon^{\tau^{s}} \cdot\left(c_{0} h^{1-n}\right)^{1-\tau^{s}}=c_{3}\left(h^{1-n}\right)^{A} \epsilon^{B} .
$$

Here $c_{3}$ depends on $\tau, s, r, M_{0}, n, R$; and $A=1-B, B=\tau^{s}$. The above inequality concludes the propagation of smallness crossing the layers from outside of the domain $\Omega$ to inside. Now we continue to propagate until the smallness reaches the neighborhood of $O$. To do this, we create the truncated cone $C(O, v, r, \vartheta)$, and apply iteratively the three spheres inequality over 
the chain of balls $B_{r_{1}}\left(w_{1}\right), B_{r_{2}}\left(w_{2}\right), \ldots, B_{r_{k(R)}}\left(w_{k(R)}\right)$ inside of the cone (see [Al-DC, proposition 3.5]). Denoted by $A_{0}=A\left(1-\tau^{k(R)-1}\right)$, we obtain

$$
\|f(y, \cdot)\|_{L^{\infty}\left(B_{r_{k(R)}}\left(w_{k(R)}\right)\right)} \leqslant \epsilon^{B \tau^{k(R)-1}} \cdot c\left(h^{1-n}\right)^{A\left(1-\tau^{k(R)-1}\right)} \leqslant \epsilon^{B \tau^{k(R)}-1} \cdot c\left(h^{1-n}\right)^{A_{0}} .
$$

For the case where we have the fixed $w \in S_{2 r}$, the proof is similar by considering $f(y, w)$ as a function of $y$. Similarly as (4.1), we have

$$
\operatorname{div}\left(A(x) \nabla_{y} f(y, w)\right)=0 \text { in } \mathcal{C} \Omega_{D}
$$

which allows us to apply the three spheres inequality iteratively over the chain of balls which are on the arc, or contained in a truncated cone. It is easy to conclude (see, [Al-DC, page 214]) that

$$
\|f(y, w)\|_{L^{\infty}\left(B_{r_{k(h)}}\left(y_{k(h)}\right)\right)} \leqslant c\left(h^{2-2 n}\right)^{\left(A \tau^{s}+1-\tau^{B}\right)\left(1-\tau^{k(h)-1}\right)} \cdot\left(\epsilon^{B \tau^{k(h)-1}}\right)^{\tau^{k(h)+B-1}} .
$$

Now by choosing $y=w=h \nu(O)$, where $\nu(O)$ is the exterior unit normal vector to $\partial \Omega_{D}$ in $O$, we obtain

$$
|f(y, y)| \leqslant c h^{A^{\prime}}\left(\epsilon^{B \tau^{k(h)-1}}\right)^{\tau^{k(h)+B-1}}
$$

where $A^{\prime}=-(2-2 n) B\left(A \tau^{s}+1-\tau^{B}\right)>0$. We observe that, for $0<h<c r$, where $0<c<1$ depends on $M_{0}$ and $k(h) \leqslant c|\log h|=-c \log h$, we write

$$
\tau^{k(h)}=\mathrm{e}^{-c \log h \log \tau}=h^{-c \log \tau}=h^{c|\log \tau|}=h^{F}
$$

with $F=c|\log \tau|$. Thus

$$
|f(y, y)| \leqslant h^{-A^{\prime}} \epsilon^{B \tau^{k(h)}}=\mathrm{e}^{-A^{\prime} \log h} \mathrm{e}^{B \tau^{k(h) \log \epsilon}}=\mathrm{e}^{-A^{\prime} \log h+B h^{F} \log \epsilon}=\frac{\epsilon^{B h^{F}}}{h^{A^{\prime}}} .
$$

The proof of proposition 3.5 is based on the asymptotic behaviour of the fundamental solutions. In the following theorem we will compare $\Gamma_{D}$ with $\Gamma_{0}$, the fundamental solution over the half space. Given a point $x=\left(x^{\prime}, x_{n}\right) \in \mathbb{R}^{n}$, let us denote $x^{*}=\left(x^{\prime},-x_{n}\right)$, and $\chi_{+}$be the characteristic function for the half-space $\mathbb{R}_{+}^{n}$. The $\Gamma_{D}$ and $\Gamma_{0}$ are defined as

$$
\begin{aligned}
\operatorname{div}\left[\left(A(x)+A(x)(k-1) \chi_{D}\right) \nabla \Gamma_{D}(\cdot, y)\right] & =-\delta(\cdot-y) \\
\operatorname{div}\left[\left(A(0)+A(0)(k-1) \chi_{0}\right) \nabla \Gamma_{0}(\cdot, y)\right] & =-\delta(\cdot-y) .
\end{aligned}
$$

When $A(0)=I$, the identity matrix, we have $\Gamma_{0}=\Gamma_{+}$, which is defined as

$$
\Gamma_{+}(x, y)= \begin{cases}\frac{1}{k} \Gamma(x, y)+\frac{(k-1)}{k(k+1)} \Gamma\left(x, y^{*}\right) & \text { for } x_{n}>0, y_{n}>0 \\ \frac{2}{k+1} \Gamma(x, y) & \text { for } x_{n} y_{n}<0 \\ \Gamma(x, y)-\frac{(k-1)}{k+1} \Gamma\left(x, y^{*}\right) & \text { for } x_{n}<0, y_{n}<0\end{cases}
$$

where $\Gamma$ is the fundamental solution for the standard Laplace operator. As for the general case $A(0) \neq I$, we refer to [Ga-Si] by performing a linear change of variable (see (4.80-4.81)) to reduce the general $A(0)$ into a simple case as $A(0)=I$. Now we have the following theorem considering only when $A(0)=I$ and $\Gamma_{0}=\Gamma_{+}$. 
Theorem 4.1. Let $\Gamma_{D}$ and $\Gamma_{0}$ be the fundamental solutions for (1.1) and (4.5), respectively. Under a priori assumption of $D$, the following estimates hold for every $x, y \in \mathbb{R}^{n}$

$$
\begin{aligned}
& \left|\Gamma_{D}(x, y)-\Gamma_{0}(x, y)\right| \leqslant \frac{C}{r_{1}^{\alpha}}|x-y|^{\alpha-n+2} \\
& \left|\nabla \Gamma_{D}(x, y)-\nabla \Gamma_{0}(x, y)\right| \leqslant \frac{C}{r_{1}^{\alpha^{2}}}|x-y|^{\alpha^{2}-n+1}
\end{aligned}
$$

where $C>0$ only depends on the a priori data and $r_{1}=\frac{r}{2} \min \left\{\frac{1}{2}\left(8 M_{0}\right)^{-1 / 2}, \frac{1}{2}\right\}$.

Proof. Let 0 be the origin of the coordinate system, $\partial D \cap B_{r}=\left\{\left(x^{\prime}, x_{n}\right) \in B_{r} \mid x_{n}=\varphi\left(x^{\prime}\right)\right\}$ where the rigid transformation $\varphi \in C^{1, \alpha}\left(\mathbb{R}^{n-1}\right)$ satisfies the definition 2.2 in which $\varphi(0)=|\nabla \varphi(0)|=0$ holds. Let $\tau \in C^{\infty}(\mathbb{R})$ be such that $\tau(t) \in[0,1]$, and $\tau(t)=1$, for $|t|<1, \tau(t)=0$, for $|t|>2$ and $\left|\frac{d \tau}{d t}\right| \leqslant 2$ for $1 \leqslant|t| \leqslant 2$. We define the change of variables $\xi=\Phi(x)$

$$
\left\{\begin{array}{l}
\xi^{\prime}=x^{\prime} \\
\xi_{n}=x_{n}-\varphi\left(x^{\prime}\right) \tau\left(\frac{\left|x^{\prime}\right|}{r_{1}}\right) \tau\left(\frac{x_{n}}{r_{1}}\right) .
\end{array}\right.
$$

It is easy to see $\Phi(\cdot)$ is a $C^{1, \alpha}$ type diffeomorphism from $\mathbb{R}^{n}$ into itself. Also, $\Phi(\cdot)$ satisfies the following properties where $c \geqslant 1$ depends only on $\alpha, M_{0}$

$$
\begin{aligned}
& \Phi\left(Q_{2 r_{1}}\right)=Q_{2 r_{1}}, \quad \Phi\left(Q_{r_{1}} \cap D\right)=Q_{r_{1}}^{+} \\
& c^{-1}\left|x_{1}-x_{2}\right| \leqslant\left|\Phi\left(x_{1}\right)-\Phi\left(x_{2}\right)\right| \leqslant c\left|x_{1}-x_{2}\right|, \quad \forall x_{1}, x_{2} \in \mathbb{R}^{n}, \\
& |\Phi(x)-x| \leqslant \frac{c}{r^{\alpha}}|x|^{1+\alpha}, \quad|D \Phi(x)-I| \leqslant \frac{c}{r^{\alpha}}|x|^{\alpha}, \quad \forall x \in \mathbb{R}^{n} .
\end{aligned}
$$

From the fundamental solution for the half space $\Gamma_{0}(x, y)$, we proceed a change of variables on (4.5) by choosing $\xi=\Phi(x), \eta=\Phi(y)$. Thus, we obtain another fundamental solution $\tilde{\Gamma}(\xi, \eta)$ such that

$$
\operatorname{div}\left[\left(1+(k-1) \chi^{+}\right) B \nabla \tilde{\Gamma}(\xi, \eta)\right]=-\delta(\xi-\eta)
$$

where $\tilde{\Gamma}(\xi, \eta)=\Gamma_{D}\left(\Phi^{-1}(\xi), \Phi^{-1}(\eta)\right), J(\xi)=(D \Phi)\left(\Phi^{-1}(\xi)\right)$ and $B(\xi)=\frac{J J^{T}}{\operatorname{det} J} A\left(\Phi^{-1}(\xi)\right)$. If we define the residual, as

$$
\tilde{R}(\xi, \eta)=\tilde{\Gamma}(\xi, \eta)-\Gamma_{0}(\xi, \eta) .
$$

Then we can solve $\tilde{R}$ by convolution with respect to $\Gamma_{0}$ and $\Gamma_{+}$, which gives

$$
\begin{array}{r}
\operatorname{div}\left(\left(1+(k-1) \chi^{+}\right) \nabla \tilde{R}(\xi, \eta)\right)=\operatorname{div}\left(\left(1+(k-1) \chi^{+}\right)(I-B) \nabla \tilde{\Gamma}(\xi, \eta)\right) \\
-\tilde{R}(\xi, \eta)=\int_{B_{\tilde{L}}}\left(1+(k-1) \chi^{+}\right)(B-I) \nabla \Gamma_{0}(\cdot, \xi) \nabla \tilde{\Gamma}(\cdot, \eta)+\tilde{C}
\end{array}
$$

here $\bar{\Omega} \subset B_{\tilde{L}}$, and $\tilde{L}>0$ depends only on the a prior data. Also the boundary term is bounded by a constant $\tilde{C}$. Now let us estimate the residual $\tilde{R}$ by considering $\xi \in Q_{r_{1} / 2}^{+}$and $\eta=e_{n} \eta_{n}$. We also separate the domain of integral into two parts $-\tilde{R}=\tilde{R}_{1}+\tilde{R}_{2}$ by using a cylinder $Q_{r_{1}}$. 


$$
\begin{array}{r}
\tilde{R}_{1}(\xi, \eta)=\int_{B_{\tilde{L}} \backslash Q_{r_{1}}}\left(1+(k-1) \chi^{+}\right)(B-I) \nabla \Gamma_{0}(\cdot, \xi) \nabla \tilde{\Gamma}(\cdot, \eta) \\
\tilde{R}_{2}(\xi, \eta)=\int_{Q_{r_{1}}}\left(1+(k-1) \chi^{+}\right)(B-I) \nabla \Gamma_{0}(\cdot, \xi) \nabla \tilde{\Gamma}(\cdot, \eta) .
\end{array}
$$

Thanks to the upper bound of the known matrix, and the ellipticity condition. We can combine Schwartz inequality with the Caccioppoli inequality, we get

$$
\left|\tilde{R}_{1}(\xi, \eta)\right| \leqslant \frac{C_{1}}{r_{1}^{2}}\left\|\Gamma_{0}(\cdot, \xi)\right\|_{L^{2}\left(\Omega \backslash Q_{3 r_{1} / 4}\right)} \cdot\|\tilde{\Gamma}(\cdot, \eta)\|_{L^{2}\left(\Omega \backslash Q_{3 r_{1} / 4}\right)}
$$

where $C_{1}=C_{1}\left(\bar{A}, \alpha, M_{0}, \lambda\right)$ is a positive constant. Moreover, we use (3.3) in [Ga-Si], the standard behavior of the Green functions at hand to obtain

$$
\left|\tilde{R}_{1}(\xi, \eta)\right| \leqslant C_{1} r_{1}^{2-n} .
$$

Now we combine (4.8) and (4.9), we have

$$
|\tilde{R}(\xi, \eta)|=\left|\tilde{R}_{1}+\tilde{R}_{2}\right| \leqslant\left|\tilde{R}_{1}\right|+\left|\tilde{R}_{2}\right| \leqslant \frac{C_{2}}{r_{1}^{\alpha}}\left(I_{1}+I_{2}\right) \leqslant \frac{C_{4}}{r_{1}^{\alpha}} h^{\alpha-n+2}
$$

where $C_{4}$ depends only on $\lambda, M_{0}, \alpha, \bar{A}, n, k$. Now our final step is to bound $R(x, y)=\Gamma_{D}(x, y)-\Gamma_{0}(x, y)$ under the original coordinate system. Arguing like in [Al-DC, proposition 3.4], we obtain

$$
\begin{aligned}
|R(x, y)| & =\left|\Gamma_{D}(x, y)-\Gamma_{0}(x, y)\right| \leqslant|\tilde{R}(\xi, \eta)|+\left|\Gamma_{0}(\Phi(x)-x, y)\right| \\
& \leqslant \frac{C_{4}}{r_{1}^{\alpha}} h^{\alpha-n+2}+\frac{C_{5}}{r_{1}^{2 \alpha}} h^{\alpha-n+2} \leqslant \frac{C_{6}}{r_{1}^{\alpha}} h^{\alpha-n+2}
\end{aligned}
$$

where $C_{6}$ depends only on $M_{0}, \alpha, n, \lambda, k, \bar{A}$. Now we prove the gradient of the residual $\nabla R(x, y)$ is also bounded from above. We start to estimate the first derivative for $\tilde{R}(\xi, \eta)$ by considering a cylinder $Q \subset B_{r_{1} / 4}^{+}(\xi)$. Let us fix $\xi \in B_{r_{1} / 4}^{+}$and $\eta_{n} \in\left(-r_{1} / 4,0\right)$ and the cylinder is defined as

$$
Q=B_{h / 8}^{\prime}\left(\xi^{\prime}\right) \times\left(\xi_{n}, \xi_{n}+\frac{h}{8}\right)
$$

Since $h=|\xi-\eta|=\left|\xi-\left(0, \eta_{n}\right)\right| \leqslant \frac{r_{1}}{2}$, we can reduce the cylinder as $Q \subset Q_{\frac{h}{4}(\xi)}$; moreover, $\xi \in \partial Q$. Then by applying [Li-Vo, theorem 1.1], we have the estimate for the semi-norm $|\nabla \tilde{\Gamma}(\xi, \eta)|_{\alpha, Q}$ by choosing $\beta=\frac{1}{2} \min \left\{\alpha, \frac{\alpha}{(\alpha+1) n}\right\}$

$$
|\nabla \tilde{\Gamma}(\xi, \eta)|_{\alpha, Q} \leqslant|\nabla \tilde{\Gamma}(\xi, \eta)|_{\beta, Q_{\frac{h}{4}(\xi)}} \leqslant C_{L} h^{-\beta-\frac{n}{2}-1}\|\nabla \tilde{\Gamma}(\xi, \eta)\|_{L^{2}\left(Q_{h / 2}(\xi)\right)}
$$

where $C_{L}$ depends only on the a priori data. Again, we use [Al-DC] proposition 3.4 (i) to obtain

$$
|\nabla \tilde{\Gamma}(\xi, \eta)|_{\alpha, Q} \leqslant C_{L} h^{-\beta-\frac{n}{2}-1} c_{1}\left(\frac{h}{2}\right)^{2-n}=C_{7} h^{-\beta-\frac{3 n}{2}+1} \leqslant C_{7} h^{\alpha-n+1}
$$

where $C_{7}$ depends only on the a priori data. By analogous argument, we could also have

$$
\left|\nabla \Gamma_{0}(\xi, \eta)\right|_{\alpha, Q} \leqslant C_{7} h^{\alpha-n+1} .
$$


Let's notice the interpolation inequality

$$
\|\nabla \tilde{R}(\xi, \eta)\|_{L^{\infty}(Q)} \leqslant C_{I}\|\tilde{R}(\xi, \eta)\|_{L^{\infty}(Q)}^{1-\delta} \cdot|\nabla \tilde{R}(\xi, \eta)|_{\alpha, Q}^{\delta}
$$

where $\delta=\frac{1}{1+\alpha}$, and $C_{I}$ depends only on $M_{0}, \alpha, \bar{A}, n$, see [Al-Si, proposition 8.3]. Since the first term of the right-hand side is already bounded in (4.10), we just need to estimate the second term $|\nabla \tilde{R}|_{\alpha, Q}^{\delta}$. By (4.11) and (4.12) and triangular inequality, we get

$$
|\nabla \tilde{R}(\xi, \eta)|_{\alpha, Q} \leqslant|\nabla \tilde{\Gamma}(\xi, \eta)|_{\alpha, Q}+\left|\nabla \Gamma_{0}(\xi, \eta)\right|_{\alpha, Q} \leqslant C_{7} h^{\alpha-n+1}
$$

Now if we plug into the interpolation inequality with (4.10), we obtain

$$
\begin{aligned}
& \|\nabla \tilde{R}(\xi, \eta)\|_{L^{\infty}(Q)} \leqslant C_{I} \frac{C_{4}^{1-\delta}}{r_{1}^{\alpha(1-\delta)}} h^{(\alpha-n+2)(1-\delta)} C_{7} h^{(\alpha-n+1) \delta} \\
& \quad=\frac{C_{8}}{r_{1}^{\tau}} h^{\tau-n+1}
\end{aligned}
$$

where $\tau=\frac{\alpha^{2}}{1+\alpha}$. Thus we use the definition of $L^{\infty}$ norm to obtain

$$
|\nabla \tilde{R}(\xi, \eta)| \leqslant \frac{C_{8}}{r_{1}^{\tau}} h^{\tau-n+1}
$$

where $C_{8}$ depends only on the a priori data. Now we consider the original coordinate system

$$
\begin{aligned}
\nabla R(x, y) & =\nabla \Gamma_{D}(x, y)-\nabla \Gamma_{0}(x, y) \\
& =\nabla \Gamma_{D}(x, y)-\nabla \Gamma_{0}(\xi, \eta)+\nabla \Gamma_{0}(\xi, \eta)-\nabla \Gamma_{0}(x, y) \\
& =\nabla \Gamma_{D}\left(\Phi^{-1}(\xi), \Phi^{-1}(\eta)\right)-\nabla \Gamma_{0}(\xi, \eta)+\nabla \Gamma_{0}(\Phi(x), \Phi(y))-\nabla \Gamma_{0}(x, y) \\
& =\nabla \tilde{R}(\xi, \eta)+\nabla \Gamma_{0}(\Phi(x), y)-\nabla \Gamma_{0}(x, y) .
\end{aligned}
$$

Concerning the absolute value of the second term on the right-hand side, we apply the the properties of diffeomorphism $\Phi(\cdot)$, as well as results from (4.6), (4.10) and (4.12), we obtain

$$
\begin{aligned}
& \left|\nabla \Gamma_{0}(\Phi(x), y)-\nabla \Gamma_{0}(x, y)\right|=\left|D \Phi(x)^{T} \nabla \Gamma_{0}(\cdot, y)_{\left.\right|_{\Phi(x)}}-\nabla \Gamma_{0}(x, y)\right| \\
& \leqslant\left|\left(D \Phi(x)^{T}-I\right) \nabla \Gamma_{0}(\cdot, y)_{\left.\right|_{\Phi(x)}}\right|+\left|\nabla \Gamma_{0}(\cdot, y)_{\left.\right|_{\Phi(x)}}-\nabla \Gamma_{0}(x, y)\right| \\
& \leqslant\left|D \Phi(x)^{T}-I\right| \cdot|| \nabla \Gamma_{0}(\cdot, y) \|_{L^{\infty}\left(Q_{r_{1}}\right)} \cdot|x-\Phi(x)| \\
& +\left|\nabla \Gamma_{0}(\cdot, y)\right|_{\alpha, Q} \cdot|\Phi(x)-x|^{\alpha} \\
& \leqslant \frac{C_{9}}{r_{1}^{\alpha^{2}}} h^{\alpha^{2}-n+1} .
\end{aligned}
$$

Now with the above estimates and (4.13), it can be concluded

$$
|\nabla R(x, y)| \leqslant|\nabla \tilde{R}(\xi, \eta)|+\left|\nabla \Gamma_{0}(\Phi(x), y)-\nabla \Gamma_{0}(x, y)\right| \leqslant \frac{C}{r_{1}^{\alpha^{2}}} h^{\alpha^{2}-n+1}
$$

where $C$ depends only on the a priori data and $r_{1}$. 
Proof of proposition 3.5. We write the upper bound of $S_{D_{1}}$ as

$$
\begin{aligned}
\left|S_{D_{1}}(y, y)\right| & =\left|(k-1) \int_{D_{1}} A(x) \nabla \Gamma_{D_{1}}(x, y) \nabla \Gamma_{D_{2}}(x, y) \mathrm{d} x\right| \\
& \geqslant C\left|\left(\int_{D_{1} \cap B_{r}(O) \cap D_{2}}+\int_{D_{1} \cap B_{\rho}(O) \cap C_{D_{2}}}\right) \nabla \Gamma_{D_{1}} \nabla \Gamma_{D_{2}}\right| \\
& -C\left|\int_{D_{1} \cap B_{r}(O) \cap \mathcal{C} B_{\rho}(O) \cap C_{D_{2}}} \nabla \Gamma_{D_{1}} \nabla \Gamma_{D_{2}}\right| \\
& -C\left|\int_{D_{1} \backslash B_{r}(O)} \nabla \Gamma_{D_{1}} \nabla \Gamma_{D_{2}}\right|
\end{aligned}
$$

where $C$ depends on $k, \bar{A}$ only, $r=|x-y|, 0<r<r_{0}, 0<\rho<\min \left\{d_{m}, r\right\}$. To explain the formula, notice we separate the integrand $\int_{D_{1} \cap B_{r}(O)} \nabla \Gamma_{D_{1}} \nabla \Gamma_{D_{2}}$ into two parts, because we do not have any information on $x$. So, either it can be $x \in D_{1} \cap B_{r}(O) \cap D_{2}$ or $x \in D_{1} \cap B_{r}(O) \cap \mathcal{C} D_{2}$. Then we separate the integrand again with respect to an even smaller ball $B_{\rho}(O)$.

If $x \in D_{1} \cap B_{r}(O) \cap D_{2}$, we use [A] lemma 3.1, which gives

$$
\nabla \Gamma_{D_{1}}(x, y) \cdot \nabla \Gamma_{D_{2}}(x, y) \geqslant C_{A}|x-y|^{2-2 n}=C_{A} r^{2-2 n}>0
$$

where $C_{A}$ depends on the a priori data. If $x \in D_{1} \cap B_{r}(O) \cap \mathcal{C} D_{2}$, we consider in a smaller ball $B_{\rho}(O)$. In this case, we actually have $x \in D_{1} \cap B_{\rho}(O) \cap \mathcal{C} D_{2}$. By definition of $d_{m}$, $B_{\rho}(O) \cap D_{2}=\emptyset$, for $x, y \in B_{\rho}(O)$, we have

$$
\left\{\begin{array}{l}
\Delta\left(\Gamma_{D_{2}}(x, y)-\Gamma(x, y)\right)=0 \text { in } B_{\rho}(O) \\
\left.\left(\Gamma_{D_{2}}(x, y)-\Gamma(x, y)\right)\right|_{\partial B_{\rho}(O)} \leqslant C_{K} \rho^{2-n}
\end{array}\right.
$$

by the maximum principle, the value on interior is smaller than boundary

$$
\left|\Gamma_{D_{2}}(x, y)-\Gamma(x, y)\right| \leqslant C_{K} \rho^{2-n} \forall x, y \in B_{\rho}(O) .
$$

And by interior gradient bound, we have

$$
\left|\nabla \Gamma_{D_{2}}(x, y)-\nabla \Gamma(x, y)\right| \leqslant C_{K_{0}} \rho^{1-n} \forall x \in B_{\rho / 2}(O) ; \forall y \in B_{\rho}(O) .
$$

Applying [A] lemma 3.1 in $B_{\rho / 2}(O)$, we have (notice $|x-y|=r>\rho$ )

$\nabla \Gamma_{D_{1}}(x, y) \cdot \nabla \Gamma_{D_{2}}(x, y) \geqslant C_{A}|x-y|^{2-2 n}-C_{K} \rho^{2-2 n}=C_{A} r^{2-2 n}-C_{K} \rho^{2-2 n}>0$.

Now we can bound the first term of (4.14) thanks to (4.15) and (4.16)

$$
\begin{aligned}
& \left|\left(\int_{D_{1} \cap B_{r}(O) \cap D_{2}}+\int_{D_{1} \cap B_{\rho}(O) \cap C_{D_{2}}}\right) \nabla \Gamma_{D_{1}} \nabla \Gamma_{D_{2}}\right| \\
\geqslant & \left|\left(\int_{D_{1} \cap B_{r}(O) \cap D_{2}}+\int_{D_{1} \cap B_{\rho}(O) \cap C_{2}}\right)\left(C_{A} r^{2-2 n}-C_{K} \rho^{2-2 n}\right)\right| \\
\geqslant & \left|\left(\int_{\left[D_{1} \cap B_{r}(O) \cap D_{2}\right] \cup\left[D_{1} \cap B_{\rho}(O) \cap C_{2}\right]}\right) c_{1} r^{2-2 n}\right| \geqslant c_{1} h^{2-n} .
\end{aligned}
$$


For the upper bounds of the second and third term, we can apply the natural bound of $\nabla \Gamma_{D_{i}}, i=1,2$. When $x \in D_{1} \cap B_{r}(O) \cap \mathcal{C} B_{\rho}(O) \cap \mathcal{C} D_{2}$, we have

$$
\begin{aligned}
& \quad\left|\int_{D_{1} \cap B_{r}(O) \cap \mathcal{C} B_{\rho}(O) \cap \mathcal{C} D_{2}} \nabla \Gamma_{D_{1}} \nabla \Gamma_{D_{2}}\right| \\
& \leqslant\left|\int_{D_{1} \cap B_{r}(O) \cap \mathcal{C} B_{\rho}(O) \cap \mathcal{C} D_{2}} c_{1}\right| x-\left.y\right|^{1-n} \cdot c_{1}|x-y|^{1-n} \mid \\
& \leqslant\left|\int_{D_{1} \cap B_{r}(O) \cap \mathcal{C} B_{\rho}(O) \cap \mathcal{C} D_{2}} c_{1} r^{1-n} \cdot c_{1} r^{1-n}\right| \\
& \leqslant c_{2} d_{m}^{2-2 n}{ }^{1-n} \int_{D_{1} \backslash B_{r}(O)} \nabla \Gamma_{D_{1}} \nabla \Gamma_{D_{2}}|\leqslant| \int_{D_{1} \backslash B_{r}(O)} c_{1}|x-y|^{1-n} \cdot c_{1}|x-y|^{1-n} \mathrm{~d} x \mid \\
& =\left|\int_{D_{1} \backslash B_{r}(O)} c_{1}^{2} r^{2-2 n} \mathrm{~d} x\right| \\
& =c_{3} .
\end{aligned}
$$

Now we can plug (4.17)-(4.19) into (4.14), we obtain the lower bound for $S_{D_{1}}(y, y)$

$$
\left|S_{D_{1}}\right| \geqslant c_{1} h^{2-n}-c_{2} d_{m}^{2-2 n}-c_{3}
$$

where $c_{i}, i=1,2,3$ depends only on the $a$ prior data.

\section{References}

[Al] Alessandrini G 1990 Singular solutions of elliptic equations and the determination of conductivity by boundary measurements J. Differ. Equ. $84252-72$

[Al-Be-Ro-Ve] Alessandrini G, Beretta E, Rosset E and Vessella S 2000 Optimal stability for inverse elliptic boundary value problems with unknown boundaries Ann. Scuola Normale Super. Pisa Cl. Sci. 29 755-806

[Al-DC] Alessandrini G and Di Cristo M 2005 Stable determanation of an inclusion by boundary measurements SIAM J. Math. Anal. 37 200-17

[Al-DC-Mo-Ro] Alessandrini G, Di Cristo M, Morassi A and Rosset E 2014 Stable determination of an inclusion in an elastic body by boundary measurements SIAM J. Math. Anal. $462692-729$

[Al-Si] Alessandrini G and Sincich E 2013 Cracks with impedance, stable determination from boundary data Indiana Univ. Math. J. 62 947-89

[DC] Di Cristo M 2007 Stable determination of an inhomogeneous inclusion by local boundary measurements J. Comput. Appl. Math. 198 414-25

[DC2] Di Cristo M 2009 Stability estimates in the inverse transmission scattering problem Inverse Problems Imaging 3 551-65

[DC-Ro] Di Cristo M and Rondi L 2003 Examples of exponential instability for inverse inclusion and scattering problems Inverse Problems 19 685-701

[DC-Ve] Di Cristo M and Vessella S 2010 Stable determination of the discontinuous conductivity coefficient of a parabolic equation SIAM J. Math. Anal. 42 183-217

[DC-Ve2] Di Cristo M and Vessella S 2011 Stability analysis of an inverse parabolic problem with discontinuous variable coefficient Proc. R. Soc. Edinburgh Sect. 141 975-99

[Ga-Si] Gaburro R and Sincich E 2015 Lipschitz stability for the inverse conductivity problem for a conformal class of anisotropic conductivities Inverse Problems 31015008 
[Is88] Isakov V 1988 On uniqueness of recovery of a discontinuous conductivity coefficient Commun. Pure Appl. Math. 41 865-77

[Kw] Kwon K 2004 Identification of anisotropic anomalous region in inverse problems Inverse Problems 20 1117-36

[Li-Vo] Li Y Y and Vogelius M 2000 Gradient estimates for solutions to divergence form elliptic equations with discontinuous coefficients Arch. Ration. Mech. Anal. $15391-151$

[Li-St-We] Littman W, Stampacchia G and Weinberger H 1963 Regular points for elliptic equations with discontinuous coefficients Ann. Scuola Normale Super. Pisa 27 43-77 